\title{
Extraction And Characterization Of Cycloartenol Isolated From Stems And Leaves of Coix Lacryma- Jobi L. And Its Potential Cytotoxic Activity
}

\section{Qiaorong Yu}

Youjiang Medical University for Nationalities

Guangbin Ye

Youjiang Medical University for Nationalities

Rong Li

Youjiang Medical University for Nationalities

Tong Li

Youjiang Medical University for Nationalities

Suoyi Huang ( $\nabla$ huangsuoyi@ymcn.edu.cn )

Youjiang Medical University for Nationalities https://orcid.org/0000-0002-6081-1601

\section{Research}

Keywords: Coix lacryma-jobi L., Cycloartenol, Extraction, Characterization, Cytotoxic

Posted Date: June 23rd, 2021

DOI: https://doi.org/10.21203/rs.3.rs-611931/v1

License: (c) (1) This work is licensed under a Creative Commons Attribution 4.0 International License. Read Full License 


\section{Abstract}

[Background] Coix lacryma-jobi L. is a nourishing food and a traditional Chinese medicine and has been used for the treatment of neuralgia, inflammatory diseases, and rheumatism. Little is known about the anti-tumor of Coix lacryma-jobi L.. In this study, the cytotoxic effects of Coix lacryma-jobi L. on HeLa, HepG2, and SGC-7901 were evaluated.

[Methods] The cytotoxic active compounds were isolated and extraction from the stems and leaves of Coix lacryma-jobi L. The structural identification of the compound was determined using ultraviolet spectroscopy, Fourier transform infrared spectroscopy and nuclear magnetic resonance spectroscopy. The cytotoxic activity in vitro effect of the compound was determined using CCK-8, Flow cytometry, and DNA Topo I inhibition experiments.

[Results] A compound F2 was isolated and purified from the petroleum ether extract of Coix lacryma-jobi L. stems and leaves. It was identified as the cycloartenol. The minimum $I C_{50}$ values of HeLa, HepG2 and SGC-7901 cells for this compound were $500,537.7$, and $336.8 \mu \mathrm{g} / \mathrm{mL}$, respectively. The compound had pro-apoptotic effects on three types of tumor cells, and had a significant inhibitory effect on DNA topoisomerase I.

[Conclusion] This study demonstrates that cycloartenol has good cytotoxic activity in vitro, suggesting that cycloartenol could be a potential candidate as a natural antitumor drug.

\section{Introduction}

Coix lacryma-jobi $\mathrm{L}$. is an annual or perennial herbaceous plant of Graminae family. It is a nourishing food and a traditional Chinese medicine and has been used for the treatment of neuralgia, inflammatory diseases, rheumatism, osteoporosis, and as a diuretic (Yang et al., 2013). The Coix lacryma-jobi L. plant has significant medicinal value in human (Xi et al., 2016). It is widely cultivated in China, Taiwan, Japan, Thailand and Korea. Coix seeds contain polysaccharides, flavonoids, phenols, proteins, fibers, vitamins and oils. Among them, the polysaccharides and polyphenols of Coix lacryma-jobi L. are considered to be the major active components and have immunomodulatory, anti-oxidant and anti-inflammatory effects (Li et al., 2019; Wang et al., 2012; Yao et al., 2015). Phenolic compounds have a variety of biological activities in antioxidant activity and xanthine oxidase inhibitor activity (Xu et al., 2017). Polysaccharides have immune potentiation effects on macrophages and should serve as a beneficial health food. Coixol shows an inhibitory action to cancer cells and a composure and demulcent action with the central nervous system, and has anti-inflammatory, health and beauty functions (Amen et al., 2017; Hu et al., 2020; Son et al., 2017).

In recent years, people are paying more attention to the pharmacological and health functions of coix seeds. For example, the coix seed constituents have strong anti-oxidant, anti-inflammatory, and antiobesity activities, and can stimulate reproductive hormones, promote uterine contraction and modulate gut microbiota (Devaraj et al., 2020). The study on the anti-tumor effect of coix seeds is in-depth, but 
there are few studies on the pharmacological effects of other parts of Coix lachryma-jobi L. Lee et al. (2008) showed that five active compounds that could inhibit tumor cells were isolated from Coix lachryma-jobi L. var. ma-yuen Stapf, and their structures and activities in vitro were characterized. All the compounds were tested for their anti-proliferative effect on A549, HT-29 and COLO205 cells. These compounds showed anti-tumor activities. Chang et al. (2018) suggested that the anti-tumor effects of the hexane fraction of the Coix lachryma-jobi L. var. ma-yuen Stapf testa ethanolic extracts on the human uterine sarcoma cancer cell line MES-SA. Manosroi et al. (2016) showed that the anti-cancer activities of Coix lachryma-jobi L. extracts on human colon adenocarcinoma in vitro. Overall, Coix lachryma-jobi L. has been used as an herbal medicine in China. Many studies of this plant have reported anti-tumor effects on human tumor cell lines.

In previous study, we observed that the aqueous and ethanol extracts of Coix lachryma-jobi L. stems and leaves could inhibit the growth of HeLa, HepG2 and SGC-7901 cell lines (Lin et al., 2018; Zhu et al., 2015). The extracts also could inhibit S180 sarcoma cells in mice, and protect the liver, spleen, thymus, and other immune organs of mice (Lin et al., 2015). Interestingly, the petroleum ether extracts of stems and leaves of Coix lachryma-jobi L. had the similar inhibitory effect on HeLa, HepG2 and SGC-7901 cell lines in vitro.

Recent studies have shown that Coix lachryma-jobi L. stems and leaves may inhibit tumor cells growth, but only a few anti-tumor specific compounds have been identified. In this study, the compound F2 from stems and leaves of Coix lachryma-jobi L. was isolated and purified. The structure of compound F2 was identified using ultraviolet (UV), Fourier transform infrared (FT-IR) and nuclear magnetic resonance (NMR) spectroscopy. Its cytotoxic activity was evaluated using CCK8, flow cytometry and DNA topoisomerase I (DNA Topo I) inhibition assay. In addition, the structure-activity relationship and action mechanism were explored, to provide a certain reference for the synthesis and development of anti-tumor drugs.

\section{Material And Methods}

\subsection{Plant and cell}

The stems and leaves of Coix lacryma-jobi L. were collected from Zubie Yao and Miao Ethnic Township in Xilin County of the Guangxi Zhuang Autonomous Region of China, in October 2017, and identified by professor Qin Daoguang from Teaching and Research Section for Ethnic Medicine of Youjiang Medical University for Nationalities as Coix lacryma-jobi L. HeLa, HepG2, and SGC-7901 cell lines were purchased from the Shanghai Cell Bank of Chinese Academy of Sciences (Shanghai, China).

\subsection{Plant material preparation and extraction}

Plant material preparation and extraction process was shown in Fig. 1. The air-dried Coix lacryma-jobi L. stems and leaves $(\sim 50 \mathrm{~kg})$ were crushed separately, and extracted three times with $200 \mathrm{~L} 95 \%$ ethanol (Chengdu Kelong Chemical Co., Ltd., Chengdu, Sichuan, China) and heated under reflux for $2 \mathrm{~h}$ (Chiang et al., 2020; Zhang et al., 2019) to yield $\sim 1 \mathrm{~kg}$ of a crude extract, which was suspended in $4 \mathrm{~L}$ ultrapure water (Milli-Q A10, Merck Millipore, Billerica, MA, USA), extracted with petroleum ether $(4 \times 10 \mathrm{~L})$ (Chengdu Kelong Chemical $\mathrm{Co}$.), and the petroleum ether extract was combined and concentrated using a rotary 
evaporator (BC-R501C, Bekai, Shanghai, China) at $40^{\circ} \mathrm{C}$ to obtain $\sim 50 \mathrm{~g}$ of petroleum ether extract. Part of the extract was separated using silica gel column chromatography (200-300 mesh, Qingdao Wave Silica Gel Desiccant Co., Ltd., Qingdao, Shandong, China). Gradient elution $(15: 1 \rightarrow 10: 1 \rightarrow 5: 1)$ was carried out with petroleum ether-ethyl acetate as the mobile phase, and the eluent was examined using thin layer chromatography (TLC) Developing agent (petroleum ether:ethyl acetate $=10: 1)(\mathrm{v} / \mathrm{v})$. The TLC plate $\left(\mathrm{GF}_{254}\right.$ plate, Qingdao Wave Silica Gel Desiccant) was sprayed using 10\% (v/v) sulfuric acid in ethanol, and then heated at $110^{\circ} \mathrm{C}$ for $5 \mathrm{~min}$. According to the TLC analysis, 11 fractions were obtained (Fr. $1-1$ to Fr. 1-11). Based on the previous experimental results (Lin et al., 2018), the Fr. 1-7 fraction (extracted from petroleum ether-ethyl acetate, 15:1) was further separated using silica gel column chromatography. The eluent was dichloromethane $\left(\mathrm{CH}_{2} \mathrm{Cl}_{2}\right)$ (Chengdu Kelong Chemical) as the mobile phase, and the eluent was again examined using TLC. According to the TLC analysis, 6 fractions were obtained (Fr. $2-1$ to Fr. 2-6). Fr. 2-2 was used for further study.

Fr. 2-2 was scanned from 190-600 nm (HPLC2695, Waters Co., Milford, MA, USA). The maximum absorptions were at 200 and $240 \mathrm{~nm}$. Fr. 2-2 was separated and purified using preparative high performance liquid chromatography (NP7060C, Hanbon Science \& Technology Co., Huaian, Jiangsu, China). The chromatographic conditions were as follows: $\mathrm{C}_{18}$ column, diameter $8 \mathrm{~cm}$, length $65 \mathrm{~cm}$. Gradient elution was carried out with methanol as the mobile phase, flow rate of $140 \mathrm{~mL} / \mathrm{min}$, wavelength of $210 \mathrm{~nm}$ and evaporative light scattering detector (ELSD-2000, Alltech, Chicago, IL, USA) to obtained the fraction Fr. $3-2\left(t_{R}=34 \mathrm{~min}\right)$. Fr. $3-2$ was concentrated at $50^{\circ} \mathrm{C}$, and crystallized with methanol-water $(100: 1, \mathrm{v} / \mathrm{v})$ to obtain the compound referred to as F2 $(250 \mathrm{mg})$.

\subsection{Structural identification of the compound F2 \\ 2.3.1 Ultraviolet (UV) analysis}

F2 (5 mg) was dissolved in $5 \mathrm{~mL}$ deionized water (Sigma-Aldrich, St. Louis, MO, USA) for scanning between 100 and $500 \mathrm{~nm}$ using a UV spectrophotometer (1260, Agilent, Palo Alto, CA, USA).

\subsubsection{Fourier-transform infrared (FT-IR) spectrum analysis}

The FT-IR spectrum analysis of the compound F2 was done using a FT-IR spectrometer (Nicolet 6700, Thermo Scientific Co., Waltham, MA, USA) at room temperature. Sample pellets were obtained by mixing the lyophilized (Alpha 1-2 LD plus, Christ, Osterode, Germany) compound F2 (5 mg) powder with $\mathrm{KBr}$ $(500 \mathrm{mg}$ ) (Sigma-Aldrich), and compressed into tablets prior for scanning in the frequency range of 400 to $4000 \mathrm{~cm}^{-1}$ and at a resolution ratio of $1 \mathrm{~cm}^{-1}$.

\subsubsection{Nuclear magnetic resonance (NMR) spectroscopy analysis}

F2 structure was measured using NMR analysis using a NMR spectrometer (Avance II-600, Bruker, Karlsruhe, Germany). F2 (25 g) was dissolved in $0.55 \mathrm{~mL}$ deuterium oxide $\left(\mathrm{D}_{2} \mathrm{O}\right)$ (Sigma-Aldrich), lyophilized, and redissolved in $\mathrm{D}_{2} \mathrm{O}$ for ${ }^{1} \mathrm{H}$ NMR $(600 \mathrm{MHz})$ and ${ }^{13} \mathrm{C} \mathrm{NMR}(150 \mathrm{MHz})$ spectrometry. All data were processed and analyzed using MestReNova software, Version 11.0.4 (Mestrelab Research, Santiago de Compostela, Spain). 


\subsection{Cell viability}

Cell viability was detected using a colorimetric Cell Counting Kit (CCK, CK04, Dojindo Laboratories, Kyushu, Japan) assay based on the manufacturer's instructions. Briefly, The tumor cells were seeded in a 96 -well plate $\left(1.0 \times 10^{4}\right.$ cells/well). The cells was cultured overnight under different treatments, the medium was exchanged with $90 \mu \mathrm{L}$ of fresh medium supplemented with $10 \mu \mathrm{L}$ CCK8 and incubated for 3 h at $37^{\circ} \mathrm{C}$. Subsequently, the absorbance was measured at $450 \mathrm{~nm}$ with a microplate reader (SpectraMax Plus 384, MD, USA). The data were obtained from 6 independent experiments.

\subsection{Flow cytometry}

Cells were digested with trypsin and then washed with phosphate-buffered saline (PBS) (Gibico). An Annexin V-FITC Apoptosis Detection Kit (AP101-100-kit, Hangzhou MultiSciences Biotech Co., Ltd., Zhejiang, China) was used to practice cell apoptosis in line with the manufacturer's instructions. The apoptotic cells were dual-stained with propidium iodide (PI) and Annexin V-FITC using an Annexin V-FITC Kit (Hangzhou MultiSciences Biotech). Analysis was carried out by Flow Cytometer (ACCURI C6, Becton, Dickinson, Franklin, NJ, USA).

\subsection{Inhibitory effect of the compound F2 on DNA Topo I}

Different concentrations of a positive control drug 10-HCPT (Selleck Chemicals, Houston, TX, USA) and different concentrations of the compound F2 were brought to $20 \mu \mathrm{L}$ of the ultrapure water system (Merck Millipore), reacted at $37^{\circ} \mathrm{C}$ for $30 \mathrm{~min}$, then $2 \mu \mathrm{L} 10 \%$ SDS was added to stop the reaction. Proteinase $\mathrm{K}(1$ $\mu \mathrm{L}$, RT403-01, Tiangen Biotech (Beijing) Co., Ltd., Beijing, China) and put in a water bath at $37^{\circ} \mathrm{C}$ for digestion for $15 \mathrm{~min}$, extracted with phenol:chloroform:isoamyl alcohol (1:1:1, v/v/v) (Sangon Biotech). The aqueous phase $(15 \mu \mathrm{L})$ was added to $3 \mu \mathrm{L}$ of $6 \times$ loading buffer, Twelve $\mu \mathrm{L}$ with the $\lambda$-Hind III digest as a marker and $1 \%$ agarose gel was used for electrophoresis (EPS-600, Tanon Science \& Technology Co., Ltd., Shanghai, China) at $5 \mathrm{~V} / \mathrm{cm}$ for $2 \mathrm{~h}$. DNA was visualized with nucleic acid stain $(10,000 \times)$ for 15 min, decolorized using ultrapure water for $15 \mathrm{~min}$, and quantification was done by a gel imaging system (Gel doc XR+, Bio-Rad, Hercules, CA, USA).

\subsection{Statistical analysis}

Statistical analysis was done with one-way analysis of variance using the Statistical Package for the Social Sciences (SPSS) software, version. 22.0 (SPSS Inc., Chicago, IL, USA). Group means were compared, using the least significant differences and $p<0.05$ or $p<0.01$ were considered to be statistically significant.

\section{Results}

\subsection{Structural analysis of the compound F2}

F2 was obtained using the extraction and purification of the petroleum ether extract from Coix lacrymajobi L. stems and leaves. F2 was a white powder. The UV absorption peak $\lambda \max =202 \mathrm{~nm}$ (Fig. 2a) suggested that there is no color-enhancing group in the molecular structure, there may be isolated double 
bonds. The FT-IR spectra (Fig. 2b) of the compound F2 shows the presence of characteristic signal peaks, such as hydroxyl groups $\left(3412 \mathrm{~cm}^{-1}\right)$, methyl groups $\left(2959,2927,2867 \mathrm{~cm}^{-1}\right)$ and double bond $(1638$ $\left.\mathrm{cm}^{-1}\right)$.

According to signals $\delta \mathrm{H} 1.60, \mathrm{~s} ; 1.684, \mathrm{~s} ; 0.887, \mathrm{~s} ; 0.808, \mathrm{~s} ; 0.959, \mathrm{~s} ; 0.962$, s in $1 \mathrm{H}-\mathrm{NMR}\left(600 \mathrm{MHz}, \mathrm{CDCl}_{3}\right)$ (Fig. 3a), there are 6 methyl signals, and $\delta \mathrm{H} 5.10, \mathrm{~m}$ is a separate double bond hydrogen signal, and $\delta \mathrm{H}$ $3.280, \mathrm{~m}$ is a carbon signal connected to oxygen atom.

On the basis of ${ }^{13} \mathrm{C}-\mathrm{NMR}\left(150 \mathrm{MHz}, \mathrm{CDCl}_{3}\right)$ and distortionless enhancement by polarization transfer (DEPT) at $135^{\circ}$ (Fig. $3 \mathrm{~b}$ ), it can be inferred that the compound has a total of 30 carbon atom signals, in which 7 are methyls $\left(\mathrm{CH}_{3}\right), 11$ methylenes $\left(\mathrm{CH}_{2}\right), 6$ methines $(\mathrm{CH})$, and 6 quaternary carbons $(\mathrm{C})$. These data inferred that there are 30 carbon atoms and 49 hydrogen atoms; and there is only one carbon signal connected to oxygen atom $\delta \mathrm{C} 78.85$, indicating that the carbon is related to hydroxyl $(\mathrm{OH})$. Therefore, it can be inferred that the molecular equation of this compound most likely was $\mathrm{C}_{30} \mathrm{H}_{50} \mathrm{O}$, it may be a triterpenoid or steroidal compound. By calculation, the unsaturation is 6 . After removing the unsaturation of a double bond, there are 5 unsaturations, so there may be 5 rings. $\delta C 125.26,130.79$ are double bond signal.

Using the carbon-hydrogen signal one to one with HSQC 2D NMR spectra (Fig. 3c), and combining $\delta \mathrm{H} 5.10, \mathrm{~m}$ and $\delta \mathrm{H} 2.041, \mathrm{ol}, \delta \mathrm{H} 2.041$, ol and $\delta \mathrm{H} 1.437$, ol, $\delta \mathrm{H} 1.437, \mathrm{ol}$ and $\delta \mathrm{H} 1.383$, ol, $\delta \mathrm{H} 1.383$, ol and $\delta H 0.879, d, J=7.3 \mathrm{~Hz}$ with COSY 2D NMR spectra (Fig. $3 d$ ) and $\delta \mathrm{C} 130.89$ and $\delta H 5.10, \mathrm{~m} ; 1.60, \mathrm{~s} ; 1.684, \mathrm{~s}$ with HMBC 2D NMR spectra (Fig. 3e), all are interrelated, so it can be inferred that there is a functional group of (1,5-dimethyl-4-ene)-hexyl, that is, 8 carbon atoms, and the other carbon atoms may be pentacyclic triterpenes or pentacyclic sterols composed of 22 carbons, including 4 methyls, and the remaining 18 carbons forming a five-ring structure. Using the similar structural data in literature (Radics et al., 1975; Teresa et al., 1987), the compound F2 was determined to be cycloartenol. The molecular weight (MW) of the compound F2 was $~ 426 \mathrm{kDa}$. The structure of compound F2 is shown in Fig. 4.

\subsection{Cell viability assay}

The cell viability of compound F2 with the HeLa, HepG2 and SGC-7901 cell lines is shown in Fig. 5 after 24,48 and $72 \mathrm{~h}$, respectively. These results showed that compound F2 inhibited the proliferation of the three cell lines at different time points. The cytotoxic active by compound F2 of the three cell lines was dose-dependent. The cell survival rate declined with the increase of drug concentration, and the cytotoxic active of the drugs was proportional to the drug concentration. At $72 \mathrm{~h}$, the $I C_{50}$ values of the compound F2 to the three tumor cells were $500,537.7$ and $336.8 \mu \mathrm{g} / \mathrm{mL}$, respectively. It can be seen that the compound F2 was sensitive to SGC-7901, and cytotoxic active was much lower than the 10-HCPT.

\subsection{Induction of apoptosis by compound F2}

From Fig. 6, it can be suggested that the apoptotic rate of the three tumor cell lines increased significantly after treatment with compound F2 and 10-HCPT. The apoptotic rates of compound F2 on HeLa, HepG2 and SGC-7901 cells were $56.67 \pm 0.24 \%, 69.09 \pm 0.55 \%$ and $67.94 \pm 1.84 \%$, respectively. It can be 
suggested that compound F2 had high pro-apoptotic effect on three tumor cell lines. Interestingly, the proapoptotic effect of compound F2 on HepG2 and SCG-7901 cells was higher than that of 10-HCPT at the same concentration. These results suggested that compound F2 could be a new promising agent for tumor treatment.

\subsection{Inhibitory effect of compound F2 on DNA Topo I}

Figure 7 shows that 10-HCPT and compound F2 of at different concentration of the positive control group had significant inhibition of the DNA Topo I, and showed a concentration-dependent effect. The inhibitory effect of compound F2 on DNA Topo I was lower than that of the positive control 10-HCPT. These results showed that they have similar effects.

\section{Discussions}

Taking the anti-tumor activity as the separation guide, a anti-tumor active compound was isolated from the stems and leaves of Coix lacryma-jobi L. Cycloartenol, a phytosterol compound, is one of the key precusor substances for biosynthesis of numerous sterol compounds. Cycloartenol has a variety of pharmacological activities such as anti-inflammatory, anti-tumor, antioxidant, antibiosis and antialzheimer's disease. Cycloartenol also plays an important role in the process of plant growth and development (Niu et al., 2018; Zhang et al., 2017). In this study, we demonstrated that cycloartenol exhibited anti-tumor activities via inhibiting suppression of cell proliferation, and induction of apoptosis in vitro.

According to the experimental results and the results of previous studies about similar chemical components or compounds, we discussed the structure-activity relationship and mechanism of antitumor effects of cycloartenol. It is inferred that the structure-activity relationship and mechanism of cycloartenol. Some studied found that extracts from stems and leaves of Coix lacryma-jobi L. have cytotoxic active. Zhu et al. (2014) used water and ethanol extracts of stems and leaves of Coix lacrymajobi L. to act on HeLa, HepG2 and SGC-7901 cells. These suggested that the water and ethanol extracts of the stems and leaves of Coix lacryma-jobi L. can inhibit these cells. Lin et al. (2018) studied the in vitro anti-tumor activity of stems and leaves of Coix lacryma-jobi L. petroleum and suggested that this compound can inhibit the growth of HeLa, HepG2 and SGC-7901 cells. On the other hand, Son et al. $(2017,2019)$ observed that Coix lacryma-jobi L. sprout extract significantly inhibited the cell proliferation in human cervical cancer HeLa and colon cancer cells in vitro. These results confirmed the cytotoxic active of cycloartenol.

The cytotoxic active of cycloartenol may be related to the spatial structure of the mother nucleus of the cycloartane, $\mathrm{C3}-\mathrm{OH}$ and double bonds (Al Muqarrabun et al., 2014; Reddy et al., 2017; Xia et al., 2018). Among the 12 cycloartane triterpenes and 4 chromones, it was suggested that two compounds showed dramatic inhibitory activities against human breast cancer cells (Li et al., 2018). In addition, Chumkaew et al. observed that a new cytotoxic sterol, stigmast-5-ene-3beta,17alpha-diol from the fruits of Syzygium 
siamense. This compound has cytotoxic activity against human oral epidermoid carcinoma, human breast cancer and human small cell lung cancer cell lines. In summary, they have similar structures.

Cycloartenol can inhibit the proliferation and promote apoptosis of HeLa cells using blocking the cells at the G2/M phase (Dai et al., 2017; Niu et al., 2018). They may inhibit the degradation, invasion and metastasis of HeLa cell-associated matrix using specifically blocking the gelatinase in HeLa cells and inhibiting the matrix metalloproteinase 2 (MMP-2), and independently promote cell apoptosis. They can also induce apoptosis from exogenous pathways using induced prostate apoptosis response-4 (Par-4) activation of downstream caspase-3, activating Fas-mediated signaling pathways. Withaferin A (3-azido Withaferin A) can inhibit ERK and Akt phosphorylation in HeLa cells, accordingly inhibiting cell proliferation and promoting apoptosis of HeLa cells (Rah et al., 2012). On the other hand, cycloartenol can inhibit the proliferation of HepG2 and promote its apoptosis. It can block cells at the G2/M phase and induced apoptosis using endogenous mitochondrial pathway, then reduce lysosomal degradation activity, accordingly leading to impaired autophagic flux of HepG2 cells, or possibly connected with regulating the expression of p53, cdc2 and caspases family proteins in the cells (Sun et al., 2015). In addition, cycloartenol can inhibit the proliferation of SGC7901 cells using blocking SGC7901 cells at the G2/M phase. It also promoted the apoptosis of SGC7901 cells using up-regulating the pro-apoptotic protein Bax and down-regulating the expression of apoptotic protein $\mathrm{Bcl}-2$, or possibly using regulating the expression of TIMP-1, TIMP-2, MMP-2, MMP-9, and CD147 to inhibit cell invasion (Niu et al., 2018). Using upregulating ATG5-ATG12 conjugate protein, it can induced autophagy, and using inhibiting $\mathrm{PI} 3 \mathrm{~K} / \mathrm{Akt} / \mathrm{mTOR} / \mathrm{p} 70 \mathrm{~S} 6 \mathrm{~K} 1$ signaling pathway, it can induced apoptosis and autophagy (Liu et al., 2011).

The 10-HCPT is a typical antineoplastic drug. As a Topo I inhibitor, it blockades the normal binding of Topo I to DNA and inhibits the rapid proliferation of tumor cells, thus exerting its antineoplastic activity (Hu et al., 2012). The inhibitory effect of compound F2 on DNA topo I activity may be related to its carbonyl, double bond, and hydroxyl groups (Huang \& Liu, 2017).

\section{Conclusions}

In summary, the cycloartenol was isolated and purified from the petroleum ether extract of Coix lacrymajobi L. stems and leaves. CCK8, flow cytometry and DNA Topo I inhibition experiments have confirmed that cycloartenol has good cytotoxic active in vitro. However, its anti-tumor molecular mechanism still needs further study.

\section{Declarations}

\section{Acknowledgements}

Not applicable.

\section{Authors' contributions}


Conceptualization, Qiaorong Yu and Suoyi Huang; Data curation, Qiaorong Yu and Tong Li; Formal analysis, Qiaorong Yu and Rong Li; Funding acquisition, Suoyi Huang; Investigation, Qiaorong Yu, Guangbin Ye, Rong Li and Tong Li; Methodology, Qiaorong Yu, Rong Li and Tong Li; Supervision, Suoyi Huang; Writing-original draft, Qiaorong Yu and Guangbin Ye; Writing-review \& editing, Guangbin Ye and Suoyi Huang. The author(s) read and approved the final manuscript.

\section{Funding}

This study was supported by the National Natural Science Foundation of China (81360684), the Guangxi Key Research and Development Program of China (AB18221095), and the Foundation for High-level Talents of Youjiang Medical University for Nationalities, China (1002018079), and the Promotion Project of Basic Ability for Young and Middle-aged Teachers in Universities of Guangxi (2020KY13007), and the Key Laboratory of Molecular Pathology (For Hepatobiliary Diseases ) of Guangxi.

\section{Availability of data and materials}

The datasets used and/or analyzed during the current study are available from the corresponding author on reasonable request.

\section{List of abbreviations}

Not applicable.

\section{Ethics approval and consent to participate}

Not applicable.

\section{Consent for publication}

Not applicable.

\section{Competing interests}

The authors declare that they have no competing interests.

\section{References}

1. Amen Y, Arung ET, Afifi MS, Halim AF, Ashour A, Fujimoto R, Goto T, Shimizu K. Melanogenesis inhibitors from Coix lacryma-jobi L. seeds in B16-F10 melanoma cells. Nat Prod Res. 2017;31(23):2712-8.

2. Al Muqarrabun LMR, Ahmat N, Aris SRS, Shamsulrijal N, Baharum SN, Ahmad R, Rosandy AR, Suratman MN, Takayama H. A new sesquiterpenoid from Scaphium macropodum (Miq.) Beumee. Nat Prod Res. 2014;28(9):597-605. 
3. Chang CC, Huang LH, Chiang WC, Hsia SM. Hexane fraction of adlay (Coix lachryma-jobi L.) testa ethanolic extract inhibits human uterine sarcoma cancer cells growth and chemosensitizes human uterine sarcoma cells to doxorubicin. Phytomedicine. 2018;47:69-80.

4. Chiang H, Lu HF, Chen JC, Chen YH, Sun HT, Huang HC, Tien HH, Huang C. (2020). Adlay Seed (Coix lacryma-jobi L.) Extracts Exhibit a Prophylactic Effect on Diet-Induced Metabolic Dysfunction and Nonalcoholic Fatty Liver Disease in Mice. Evidence-Based Complementary and Alternative Medicine, 2020, 9519625.

5. Chumkaew P, Kato S, Chantrapromma K. New cytotoxic steroids from the fruits of Syzygium siamense. J Asian Nat Prod Res. 2010;12(5):424-8.

6. Dai L, Wang G, Pan WS. (2017). Andrographolide Inhibits Proliferation and Metastasis of SGC7901 Gastric Cancer Cells. Biomed Research International, 2017, 6242103.

7. Hu YS, Zhou QY, Liu TL, Liu ZJ. Coixol Suppresses NF-kB, MAPK Pathways and NLRP3 Inflammasome Activation in Lipopolysaccharide-Induced RAW 264.7 Cells. Molecules. 2020;25(4):894.

8. Hu WJ, Hu W, Fang Y. Research Progress on anti-tumor mechanism of Camptothecin. China Pharmacy. 2012;23(27):2581-4.

9. Huang YL, Liu Y. Preliminary Anti-tumor Activity Study of Flavonoids from Extracts of Rosa laevigata. Genomics Applied Biology. 2017;36(10):4007-11.

10. Li YL, Tian XD, Li SC, Chang LJ, Sun P, Lu YB, Yu XY, Chen SW, Wu ZQ, Xu Z, Kang WR. Total polysaccharides of adlay bran (Coix lachryma-jobi L.) improve TNF-a induced epithelial barrier dysfunction in Caco-2 cells via inhibition of the inflammatory response. Food Funct. 2019;10(5):2906-13.

11. Lee MY, Lin HY, Cheng FW, Chiang WC, Kuo YH. Isolation and characterization of new lactam compounds that inhibit lung and colon cancer cells from adlay (Coix lachryma-jobi L. var. ma-yuen Stapf) bran. Food Chem Toxicol. 2008;46(6):1933-9.

12. Lin Y, Lu SH, Yu QR, Huang SY. Study on the anti-tumor activity of petroleum ether fractions of the extract from stems and leaves of coix in vitro. Chinese Journal of Clinical Pharmacology. 2018;34(3):282-4. (in Chinese).

13. Lin Y, Li J, Qin YC, Zhu XY, Huang SY. Study on antitumor effects of coix leaves in vivo. Chinese Journal of Hospital Pharmacy. 2015;35(15):1357-9. (in Chinese).

14. Li X, Wang W, Fan Y, Wei Y, Yu LQ, Wei JF, Wang YF. Anticancer efficiency of cycloartane triterpenoid derivatives isolated from Cimicifuga yunnanensis Hsiao on triple-negative breast cancer cells. Cancer Management Research. 2018;10:6715-29.

15. Liu J, Zhang Y, Qu JL, Xu L, Hou KZ, Zhang JD, Qu XJ, Liu YP. $\beta$-Elemene-induced autophagy protects human gastric cancer cells from undergoing apoptosis. BMC Cancer. 2011;11:183.

16. Manosroi A, Sainakham M, Chankhampan C, Manosroi W, Manosroi J. In vitro anti-cancer activities of Job's tears (Coix lachryma-jobi Linn.) extracts on human colon adenocarcinoma. Saudi Journal of Biological Science. 2016;23(2):248-56. 
17. Niu HF, Li XM, Yang AJ, Jin ZZ, Wang XN, Wang Q, Yu CN, Wei ZF, Dou CY. Cycloartenol exerts antiproliferative effects on Glioma U87 cells via induction of cell cycle arrest and p38 MAPK-mediated apoptosis. Journal of BUON. 2018;23(6):1840-5.

18. Radics L, Peredy MK, Corsano S, Standoli L. Carbon-13 NMR spectra of some polycyclic triterpenoids. Tetrahedron Lett. 1975;16(48):4287-90.

19. Reddy SD, Siva B, Babu VSP, Vijaya M, Nayak VL, Mandal R, Tiwari AK, Shashikala P, Babu KS. New cycloartane type-triterpenoids from the areal parts of Caragana sukiensis and their biological activities. Eur J Med Chem. 2017;136:74-84.

20. Rah B, Amin H, Yousuf K, Khan S, Jamwal G, Mukherjee D, Goswami A. A Novel MMP-2 Inhibitor 3azidowithaferin A (3-azidoWA) Abrogates Cancer Cell Invasion and Angiogenesis by Modulating Extracellular Par-4. PLoS One. 2012;7(9):e44039.

21. Son ES, Kim YO, Park CG, Park KH, Jeong SH, Park JW, Kim SH. Coix lacryma-jobi var. ma-yuen Stapf sprout extract has anti-metastatic activity in colon cancer cells in vitro. BMC Complementary Alternative Medicine. 2017;17(1):486-98.

22. Sun HY, Liu BB, Chen SB. Isolation and structure elucidation of cycloartane triterpenes from Cimicifuga foetida L. and their anticancer activities. Central South Pharmacy. 2015;13(03):234-8.

23. Teresa DP, Urones JG, Marcos IS, Basabe P, Cuadrado MJS, Moro RF. Triterpenes from Euphorbia Broteri. Phytochemistry. 1987;26(6):1767-76.

24. Wang L, Sun J, Yi Q, Wang X, Ju X. Protective effect of polyphenols extract of adlay (Coix lachrymajobi L. var. ma-yuen Stapf) on hypercholesterolemia-induced oxidative stress in rats. Molecules. 2012;17(8):8886-97.

25. Xi XJ, Zhu YG, Tong YP, Yang XL, Tang NN, Ma SM, Li S, Cheng Z. Assessment of the Genetic Diversity of Different Job's Tears (Coix lacryma-jobi L.) Accessions and the Active Composition and Anticancer Effect of Its Seed Oil. PLoS One. 2016;11(4):e0153269.

26. Xu L, Wang P, Ali B, Yang N, Chen YS, Wu FF, Xu XM. Changes of the phenolic compounds and antioxidant activities in germinated adlay seeds. Journal of the Science Food Agriculture. 2017;97(12):4227-34.

27. Xia HM, Dai YP, Sun LL. Research progress on cycloartane triterpenoids of Actaea. China Journal of Chinese Materia Medica. 2018;43(20):4000-10. (in Chinese).

28. Yang RS, Lu YH, Chiang WC, Liu SH. Osteoporosis Prevention by Adlay (Yì Yi: The Seeds of Coix Lachryma-Jobi L. var. ma-yuen Stapf) in a Mouse Model. Journal of Traditional Complementary Medicine. 2013;3(2):134-8.

29. Yao Y, Zhu YY, Gao Y, Ren G, X. Effect of ultrasonic treatment on immunological activities of polysaccharides from adlay. Int J Biol Macromol. 2015;80:246-52.

30. Zhu XY, Lin Y, Huang SY, Yu S. Inhibitory effects of Adlay extracts from stems and leaves on tumor cells. Food Research Development. 2015;36(21):1-3. (in Chinese).

31. Zhang CF, Zhang WF, Shi RY, Tang BY, Xie SC. Coix lachryma-jobi extract ameliorates inflammation and oxidative stress in a complete Freund's adjuvant-induced rheumatoid arthritis model. Pharm Biol. 
2019;57(1):792-8.

32. Zhang ZL, Luo ZL, Shi HW, Zhang LX, Ma XJ. Research advance of functional plant pharmaceutical cycloartenol about pharmacological and physiological activity. China Journal of Chinese Materia Medica. 2017;42(3):433-7. (in Chinese).

\section{Figures}

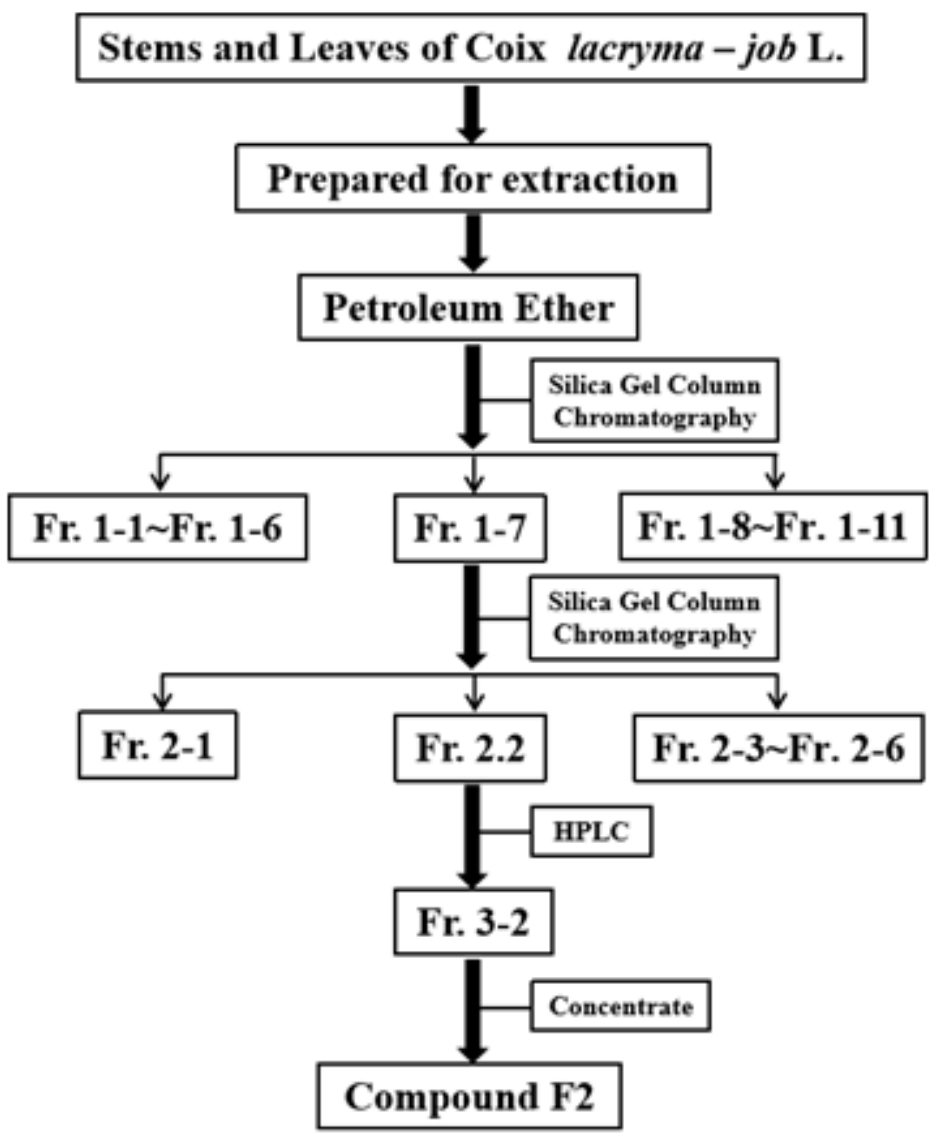

Figure 1

Flow chart showing plant material preparation and extraction process.
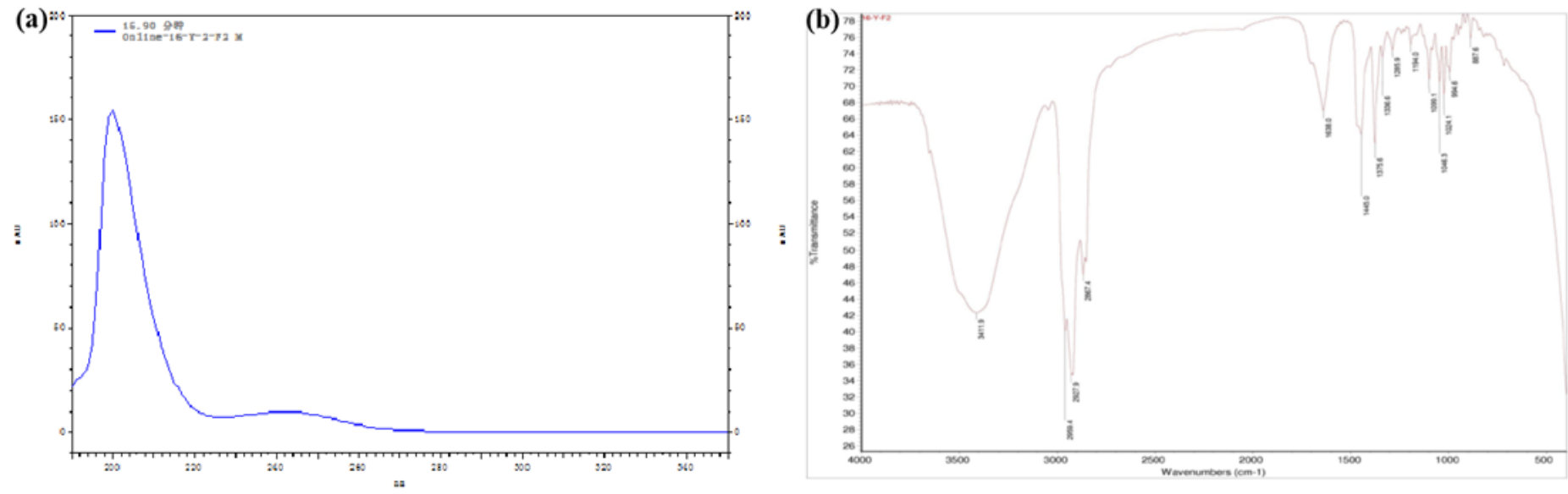
Figure 2

UV (a), and FT-IR (b) chromatogram of the compound F2 from Coix lacryma-jobi L. stems and leaves.

(a)

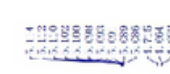

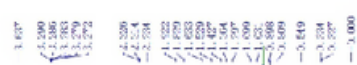

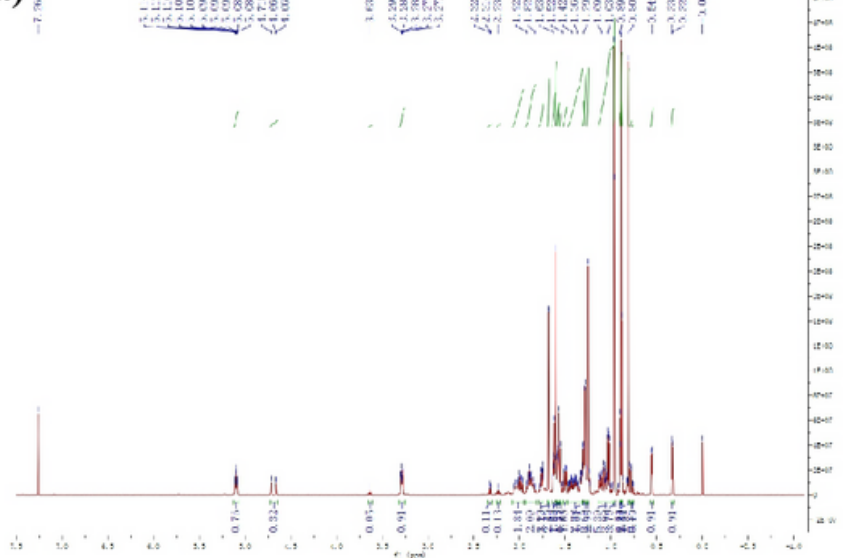

(c)
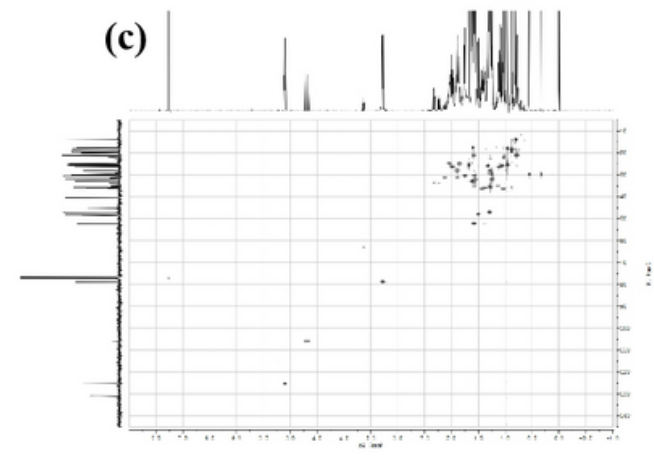

(d) (b)
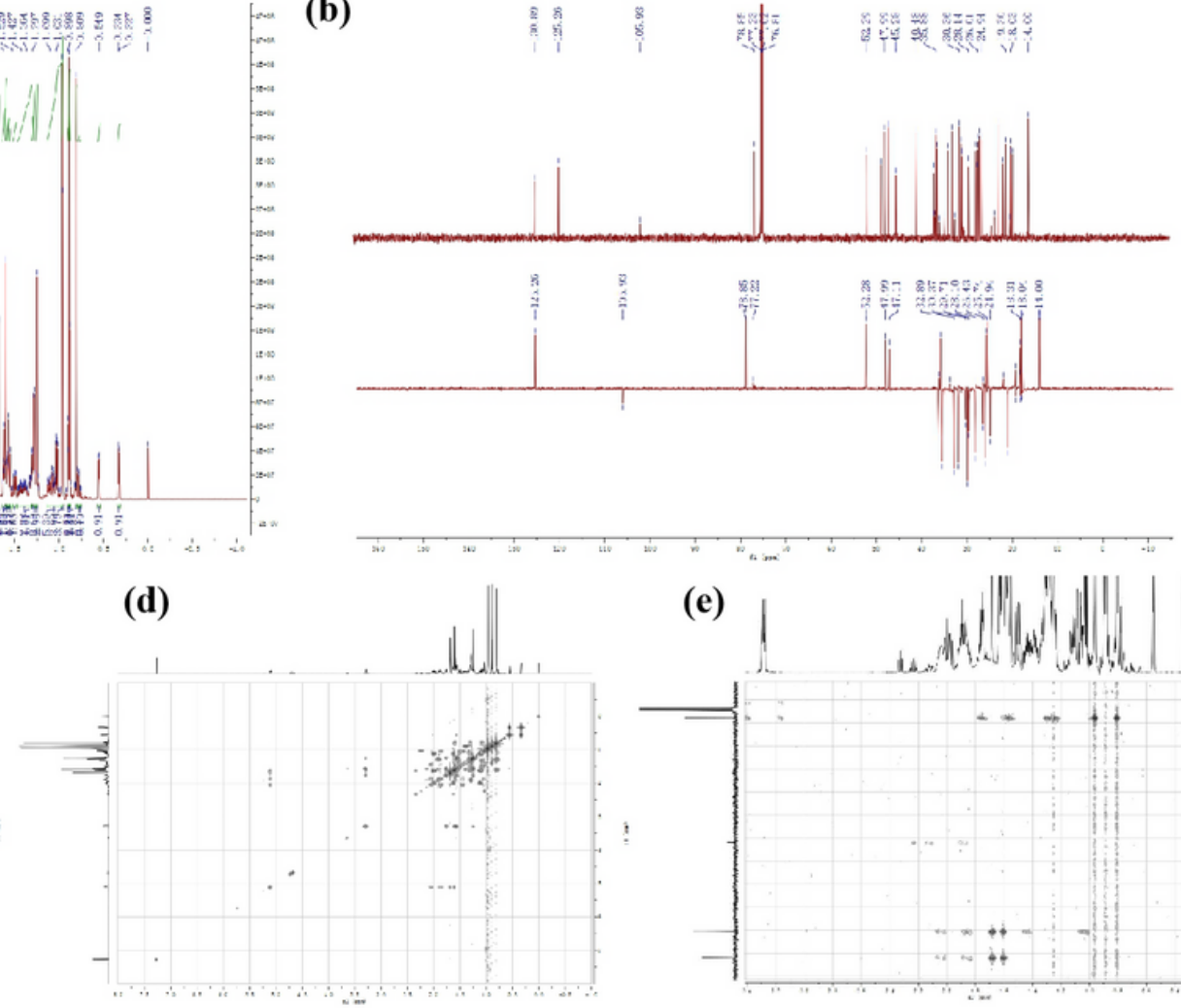

(e)
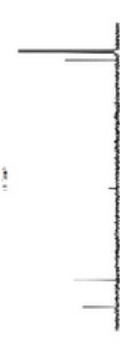

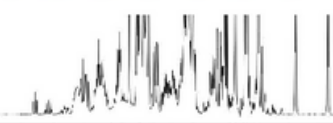

lasing $x$
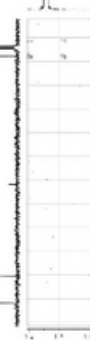

\section{Figure 3}

1D and 2D NMR spectra of compound F2 from Coix lacryma-jobi L. stems and leaves. (a) 1H- NMR; (b) 13C- NMR; (c) HSQC 2D NMR; (d) COSY 2D NMR; (e) HMBC 2D NMR. 


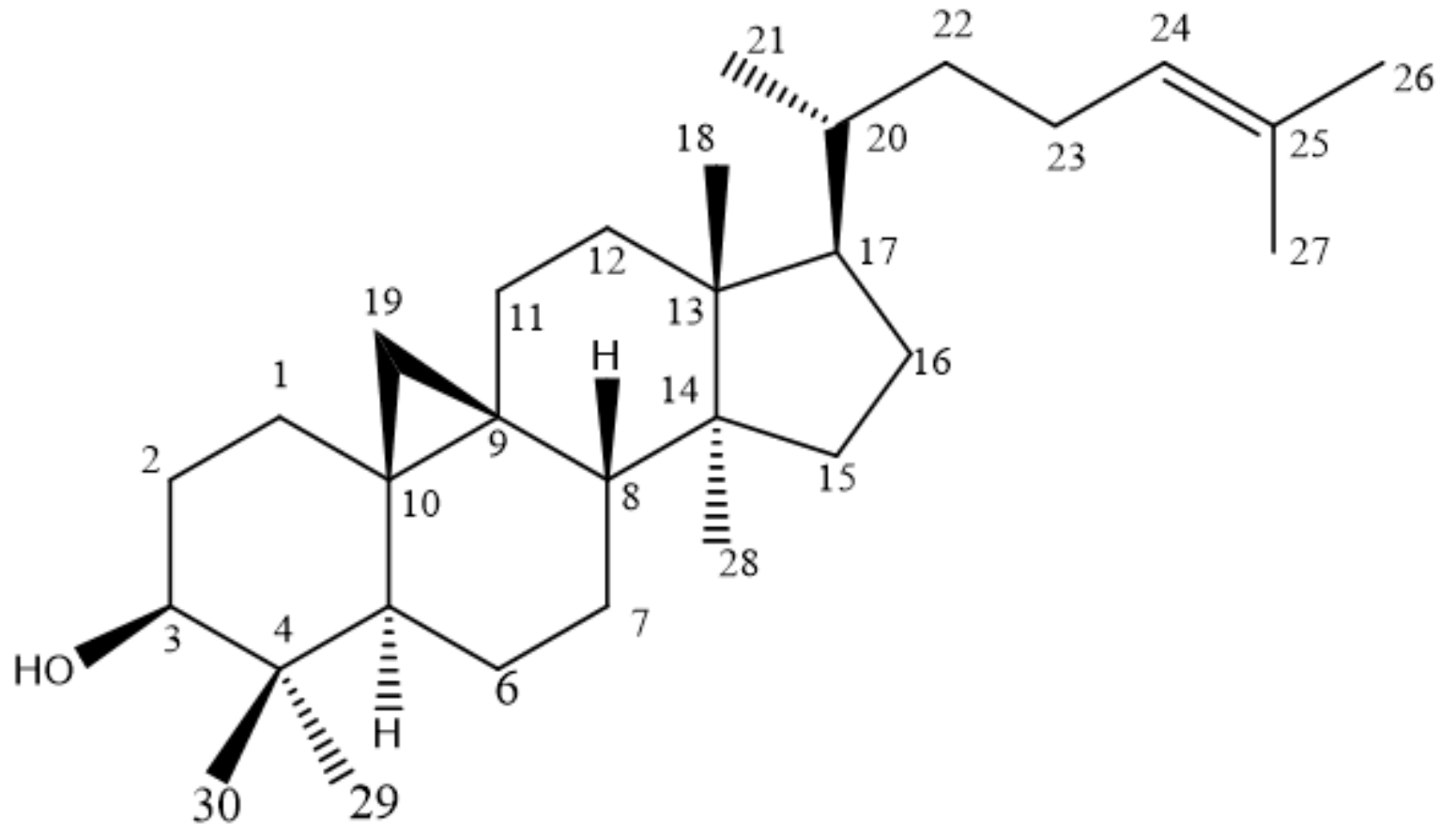

Figure 4

The structure of the compound F2. 


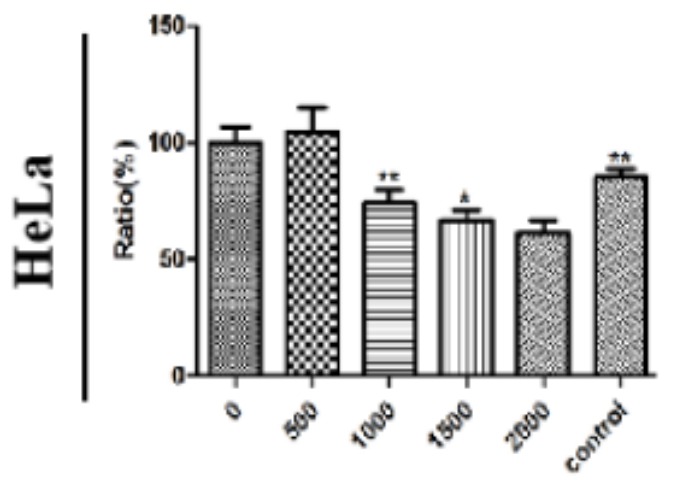

Concentration $(\mu \mathrm{g} / \mathrm{mL})$

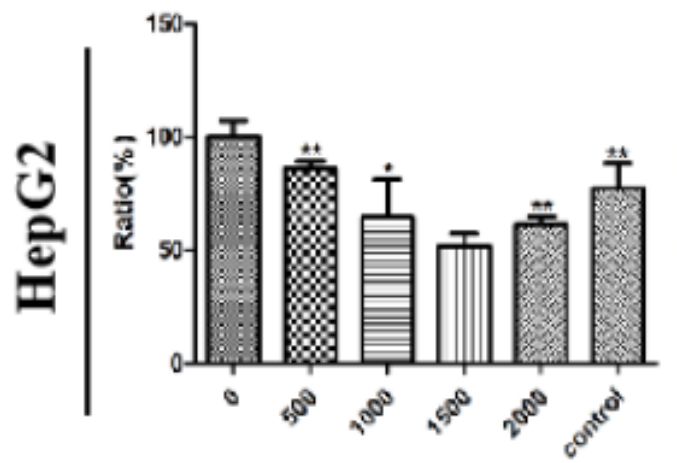

Concentration( $\mu g / \mathrm{mL}$ )

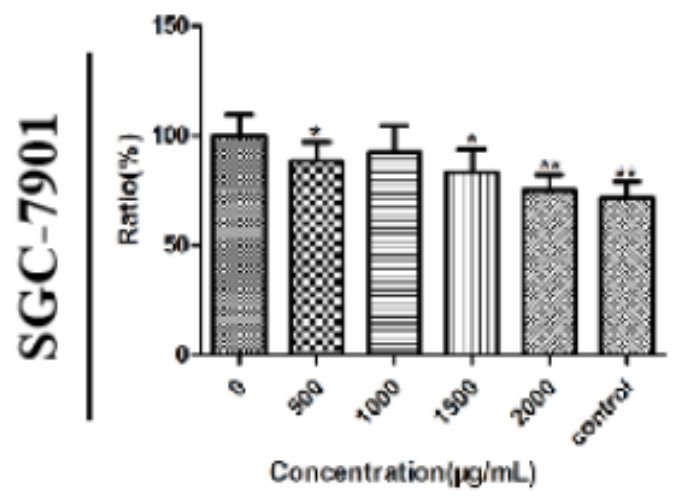

$24 \mathrm{~h}$

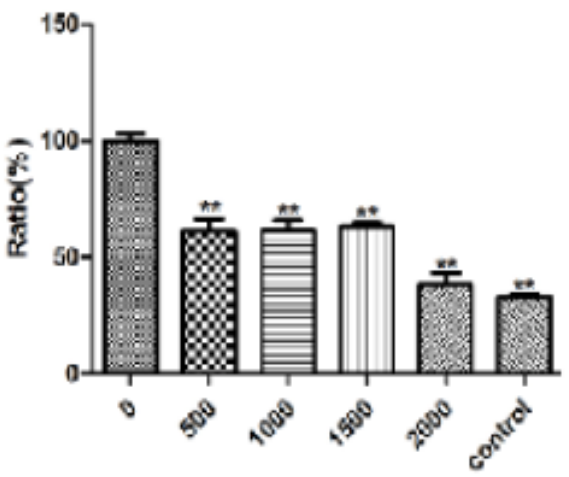

Concentration(jg/mL)

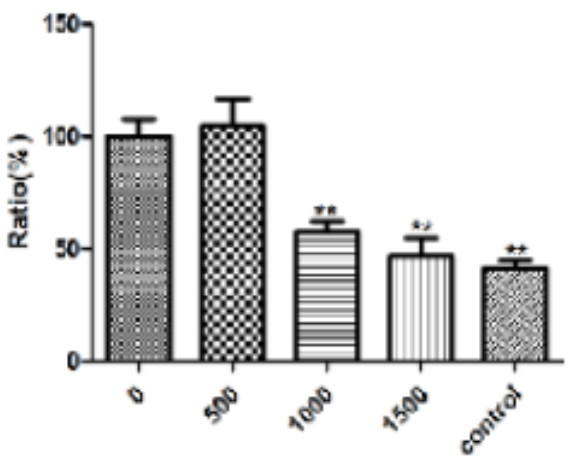

Coneentration(rgimL)

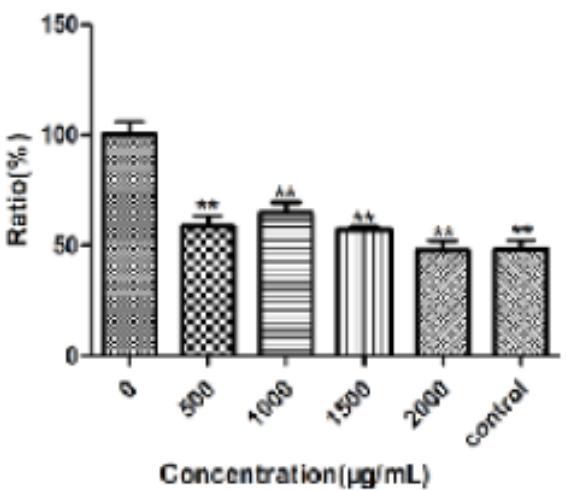

$48 \mathrm{~h}$

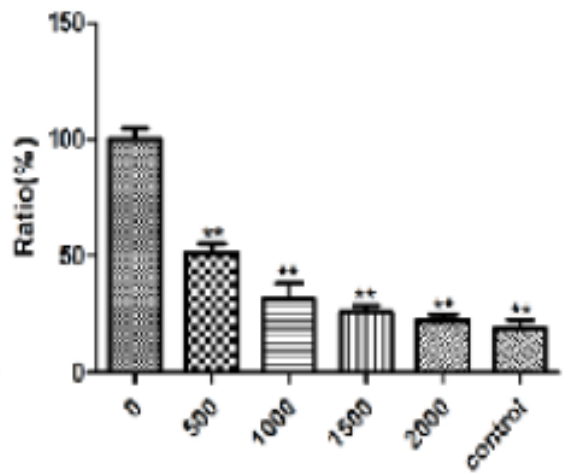

Concentration $\left(1 \mathbf{g}^{\prime} / \mathrm{mL}\right)$

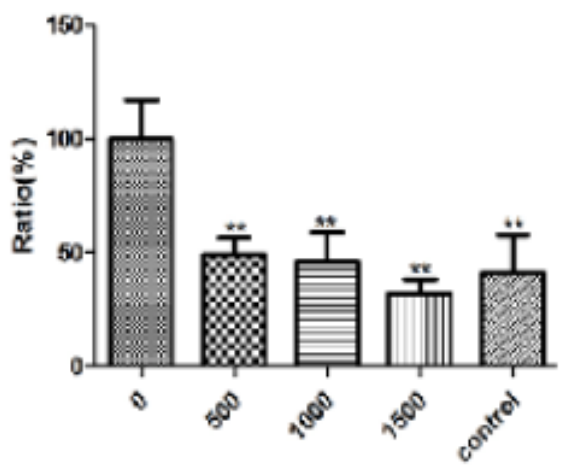

Concentration ( $\mu \mathrm{g} / \mathrm{mL}$ )

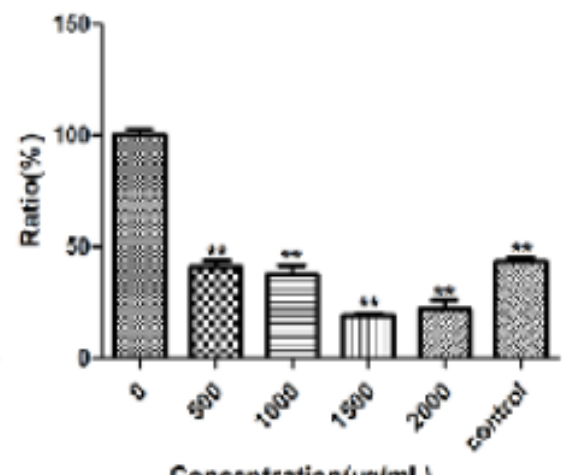

Concentration( $\mathrm{\mu g} / \mathrm{mL}$ )

$72 \mathrm{~h}$

Figure 5

Effect of compound F2 on the viability of HeLa, HepG2 and SGC-7901 cell lines. The cell activity of compound F2 treatment for 24,48 and $72 \mathrm{~h}$, respectively. Compared with no treatment group, $\star * ; p<0.01$, *; p<0.05, control; 10 -HCPT. 
(a)

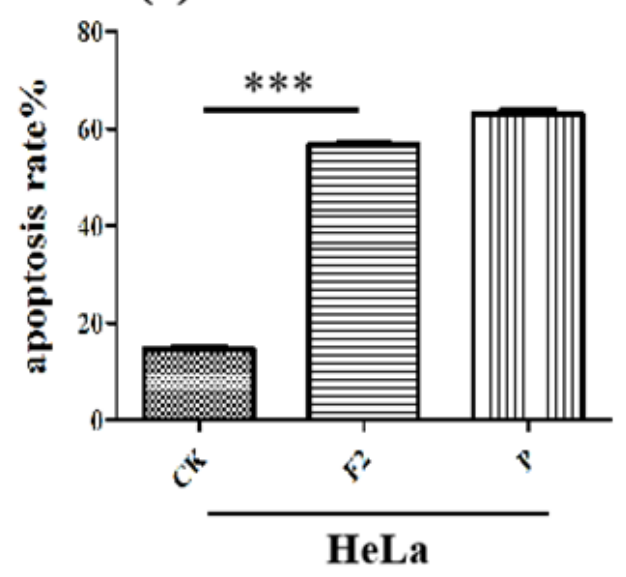

(b)

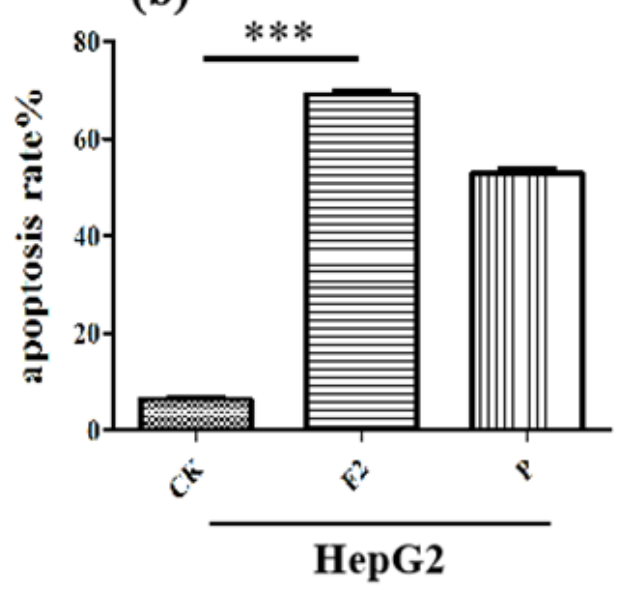

(c)

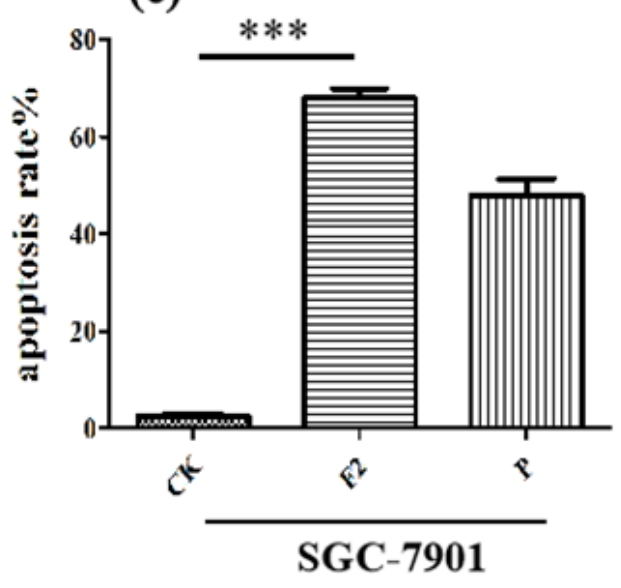

Figure 6

Apoptotic effect of compound F2 on HeLa (a), HepG2 (b) and SGC-7901 (c) cell lines. Bar charts depicting percentage of apoptotic rate for the treatments on the corresponding cell lines. Values are mean \pm SD $(n=3)$. CK, control; P, 10-HCPT; ***, $p<0.01$.

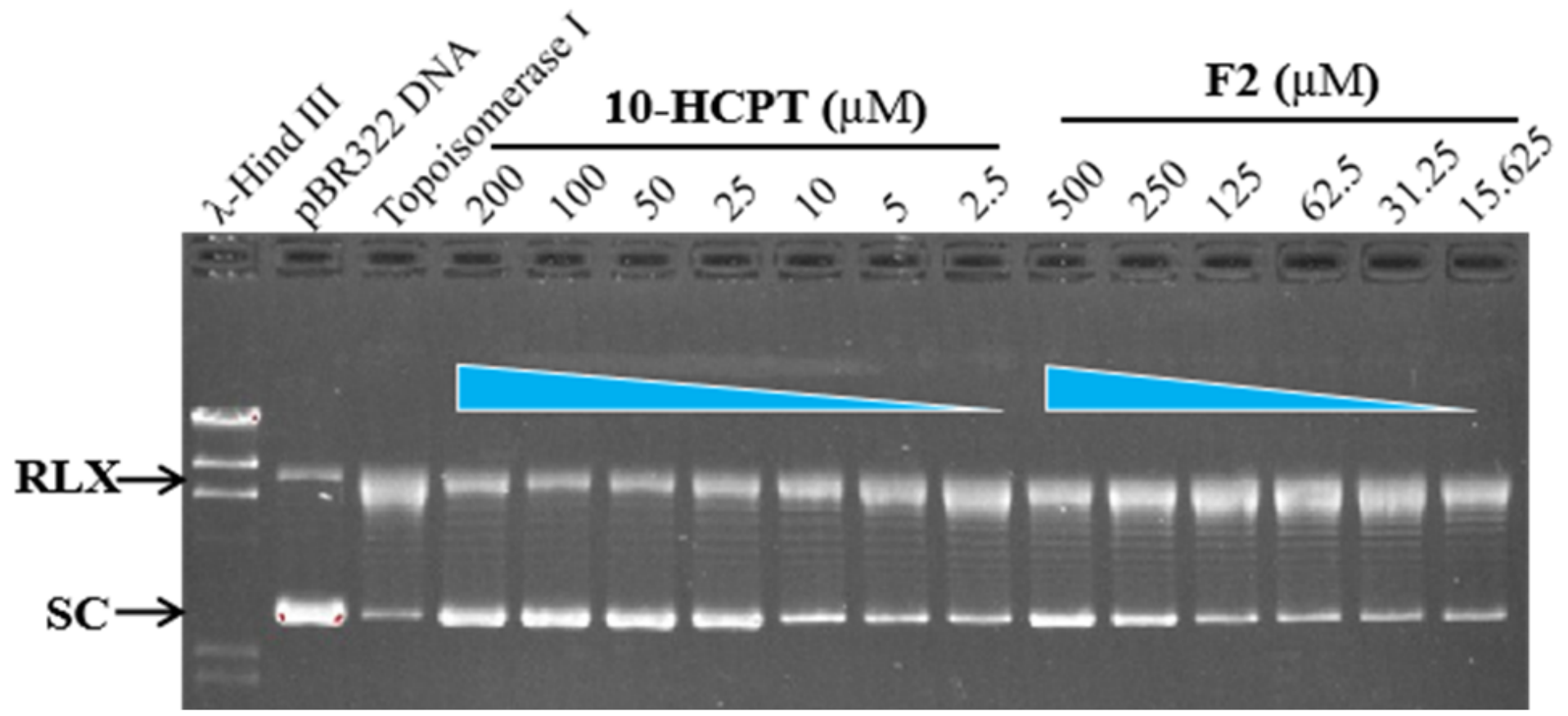

Figure 7

Effect of compound F2 on DNA Topo I. From left to right: the first lane is $\lambda$-Hind III digest as a marker; the second lane is pBR322 DNA alone; the third lane is pBR322 DNA and DNA Topo I, but no compound F2; the fourth to tenth lanes are 10-HCPT with final concentration of $200 \sim 2.5 \mu \mathrm{M}$; the eleventh to sixteenth lanes are compound F2 with a final concentration of $500 \sim 15.63 \mu \mathrm{M}$; SC is a supercoiled plasmid and $\mathrm{RLX}$ is relaxed DNA. 